\title{
Unmasking a role for sex chromosomes in gene silencing
}

Danielle M Maatouk and Blanche Capel*

\begin{abstract}
Several sexually dimorphic phenotypes correlate with sex-chromosome dosage rather than with phenotypic sex. New research suggests that sex chromosome dimorphism helps to regulate gene silencing.
\end{abstract}

During mammalian development, sexual development is initiated in the embryonic gonad. Male gonadal cells express the Sry gene (sex determining region Y), a Ylinked gene which triggers differentiation of the testis. Steroidogenic Leydig cells, within the testis, produce testosterone, masculinizing non-gonadal tissues including the sex ducts, the external genitalia and parts of the brain. The dominant role of hormones in masculinizing the brain was shown about 50 years ago by Alfred Jost. By treating female guinea pigs with testosterone prenatally, he demonstrated irreversible male-type behavioral changes. This early research suggested that $\mathrm{XX}$ and $\mathrm{XY}$ cells were equivalent and that sexual dimorphisms resulted from exposure to male or female gonadal hormones [1]. However, the question of whether sexual dimorphism is completely reliant on hormones or whether there is also a genetic component has remained an area of interest.

Heteromorphic sex chromosomes make cells in the male and female intrinsically different in terms of the dose of sex-linked genes (as the female has two $\mathrm{X}$ chromosomes and the male has an $\mathrm{X}$ and a $\mathrm{Y}$ chromosome). This difference is balanced in part by inactivating one $\mathrm{X}$-chromosome in females, which equalizes $\mathrm{X}$ gene dosage between males and females. However, in mice and humans, many genes escape inactivation, increasing the gene dosage of these loci in XX cells [2]. Therefore, it is not inconceivable that intrinsic chromosomal differences affect the differentiation of XX and XY cells. Brain and

*Correspondence: b.capel@cellbio.duke.edu

Department of Cell Biology, Duke University Medical Center, Durham, NC 27710, USA nerve cells seem to be sensitive to this inherent difference in gene dosage. For example, in cultures of mouse midbrains, where the effect of hormones is probably negligible, XY explants develop more dopamine neurons than do XX explants. Similarly, the density of vasopressin fibers correlates with the presence of the $\mathrm{Y}$ chromosome, and not with phenotypic sex (female or male) [3]. However, Sry expression has been reported in regions of the mouse brain, challenging the idea that differences in neuronal tissue are controlled by chromosome dosage effects [4].

Outside of the nervous system, sexually dimorphic characteristics exist that precede gonad differentiation, and hence hormone production [1]. In several species, the size of male and female embryos differ. In the marsupial, differentiation of the scrotum and pouch begin before the gonad forms and is controlled by Xchromosome dosage. These examples support the idea that sex chromosome differences affect male and female development. However, it has been challenging to study sexual differentiation free from the effects of hormones to assess broader contributions of dosage effects on sexual dimorphisms. A recent study by Wijchers et al. [5] has succeeded in doing this.

\section{Sexual dimorphism and sex chromosome complement}

In a recent Developmental Cell paper, Wijchers et al. [5] used the 'four core genotypes' (FCG) mouse model to separate the genetic versus hormone dependent effects on variable silencing of heterochromatin. This mouse model was originally designed to test the relative contribution of sex chromosomes and phenotypic sex to sexually dimorphic characteristics [6]. In this system, $\mathrm{XX}$ and $\mathrm{XY}^{-}$ mice are phenotypic females ( $\mathrm{Y}^{-}$denotes a deletion of $\left.S r y\right)$, although they have different sex chromosome compositions. Similarly, XY-Sry and XXSry are phenotypic males exposed to similar levels of androgens, although they have different sex chromosome compositions.

Wijchers et al. [5] show that in a mouse model for position effect variegation, expression of a transgene, human $C D 2(h C D 2)$, which has integrated into a heterochromatic region and undergoes variable silencing in 
$\mathrm{T}$ cells, is more strongly silenced in males (XY) than in females (XX). To determine whether the dimorphic gene silencing was caused by a genetic or phenotypic component, they crossed the $h C D 2$ transgene onto the FCG mice. They found that $\mathrm{XY}^{-}$females and $\mathrm{XY}^{-}$Sry males exhibited increased transgene silencing compared with XX females and XXSry males. Therefore, silencing did not depend on whether the individual was male or female, but was dependent on a repressive effect of the $Y$ chromosome, or a role for two $\mathrm{X}$ chromosomes in increasing expression. To distinguish between these two possibilities, they used two additional mouse lines; $\mathrm{XX}^{\mathrm{Y}^{s}}$ males (where a $Y^{*}$ is attached to an $\mathrm{X}$ chromosome) and $\mathrm{XO}$ females (which carry only one $\mathrm{X}$ chromosome). They found that $\mathrm{XO}$ females had lower expression of the $h C D 2$ transgene than did $\mathrm{XX}^{\mathrm{Y}^{*}}$ males. Although this experiment is confounded by different hormone environments, the positive effect of two $\mathrm{X}$ chromosomes on transgene expression seemed to be dominant over any repressive effect the $\mathrm{Y}$ chromosome might have.

Having uncovered a sex-chromosome effect on gene silencing, the authors [5] performed a genome wide study to identify endogenous autosomal genes sensitive to sex chromosome composition. Microarray analyses of gene expression from FCG thymus samples identified over 2,000 affected genes, including an overlapping set of 369 genes that were differentially expressed between phenotypic males of different sex chromosome constitutions (XXSry versus XY'Sry), and between phenotypic females of different sex chromosome constitutions (XX versus $\mathrm{XY}^{-}$), termed the 'sex-chromosome-complement sensitive set' (SCS). Because the $h C D 2$ transgene is silenced by the heterochromatin protein $\mathrm{HP} 1$, Wijchers and co-workers [5] tested the SCS gene set for sensitivity to HP1 and found that relative to the number of genes in the entire T-cell genome silenced by this factor (4\%), the SCS gene set was enriched for HP1-silenced genes (12\%). However, in contrast to the results obtained using the CD2 transgene reporter, $\mathrm{X}$-chromosome dosage was not the dominant force in silencing the SCS genes. Instead, they found that XX females in fact exhibited more silencing than $X^{-}$females. Here, it seemed that either the increased $\mathrm{X}$ dosage was repressive, or the $\mathrm{Y}$ chromosome had a positive influence on expression of the SCS genes.

One of the most significant differences in the expression levels of these genes was seen between XY-Sry males and XY-females. Here, the sex-chromosome dosage was identical, but the hormonal environment was different. When the comparison was repeated between $\mathrm{XY}$ Sry males and XX females (recapitulating a normal chromosome complement), the expression differences were reduced. The authors [5] conclude that the SCS genes respond to multiple sex chromosome inputs, some of which are distinct from the effects on variegation.
Overall Sry, and/or the hormonal environment it induces, tends to have a role in balancing the effects of sex chromosome dosage.

Finally, Wijchers et al. [5] investigated whether the SCS genes constitute a subset of genes that normally show expression differences between males and females. They identified 1,751 genes that were dimorphically expressed in the thymus of XY males and XX females, $10 \%$ of these (175 genes) overlapped the SCS gene set, whereas an additional $23 \%$ were sensitive in only one sex. The authors [5] conclude that a large portion (about 30\%) of the genes that are normally differentially expressed in male and female thymus can be attributed to effects of sex chromosome composition.

\section{Future perspectives}

Prior to this study, the effects of sex chromosomes on sexual dimorphisms were mostly limited to morphological observations, most of which were restricted to differences in brain structure and nervous system differentiation. This study provides the first evidence that sex chromosomes can influence much broader, genomewide variations.

How might this work? The authors point out [5] that $\mathrm{Xu}$ et al. $[7,8]$ recently used the same FCG mouse model to establish that increased expression of the two X-linked histone demethylases, Jarid1c and Utx, was dependent on having two copies of the $\mathrm{X}$ chromosome, and not on sexual differentiation. Higher expression of chromatin modifiers in one sex could directly affect the expression level of large numbers of genes, leading to sexually dimorphic gene expression and morphological phenotypes. In addition, it has been proposed for Drosophila that a large heterochromatinized region, such as the inactive $\mathrm{X}$, could act as a sink to sequester inactivating factors reducing access for other silenced regions [9]. Along these lines, in Caenorhabditis elegans, dosage compensation proteins not only control X chromosome expression levels, but also act in other complexes that affect other regions of the genome, creating interdependent mechanisms [10].

In humans, males and females exhibit sexually dimorphic changes that not only lead to morphological, physiological, and behavioral differences, but also to differences in disease susceptibility. The results presented here [5] suggest that the normal XX and XY karyotypes function to equalize gene expression in the context of their respective hormone environments. Disrupting the genetic component of this balance can lead to expression changes in genes such as the SCS set, which appear to be sensitized to differences in sex-chromosome dosage. Human patients with sex chromosome aneuploidy, such as individuals with Turner's (XO females) or Klinefelter's (XXY males) syndromes, can have gene expression 
differences that result from this imbalance. These differences can contribute to the distinct social and cognitive differences that distinguish these individuals from their 'normal' female and male counterparts [3]. It will be interesting to see if the SCS genes identified by Wijchers et al. [5] are also dimorphically expressed in other cell types, or if different sets of genes are sensitive to sex chromosome dosage in other cell lineages.

Published: 27 September 2010

\section{References}

1. Arnold AP, Xu J, Grisham W, Chen X, Kim YH, Itoh Y: Minireview: Sex chromosomes and brain sexual differentiation. Endocrinology 2004, 145:1057-1062.

2. Carrel L, Willard HF: $X$-inactivation profile reveals extensive variability in X-linked gene expression in females. Nature 2005, 434:400-404.

3. Arnold AP, Burgoyne PS: Are XX and XY brain cells intrinsically different? Trends Endocrinol Metab 2004, 15:6-11

4. Dewing P, Chiang CW, Sinchak K, Sim H, Fernagut PO, Kelly S, Chesselet MF, Micevych PE, Albrecht $K H$, Harley VR, Vilain E: Direct regulation of adult brain function by the male-specific factor SRY. Curr Biol 2006, 16:415-420.
5. Wijchers PJ, Yandim C, Panousopoulou E, Ahmad M, Harker N, Saveliev A Burgoyne PS, Festenstein R: Sexual dimorphism in mammalian autosomal gene regulation is determined not only by Sry but by sex chromosome complement as well. Dev Cell 2010, 19:477-485.

6. De Vries GJ, Rissman EF, Simerly RB, Yang LY, Scordalakes EM, Auger CJ, Swain A, Lovell-Badge R, Burgoyne PS, Arnold AP: A model system for study of sex chromosome effects on sexually dimorphic neural and behavioral traits. J Neurosci 2002, 22:9005-9014.

7. Xu J, Deng X, Disteche CM: Sex-specific expression of the X-linked histone demethylase gene Jarid1c in brain. PLoS One 2008, 3:e2553.

8. Xu J, Deng X, Watkins R, Disteche CM: Sex-specific differences in expression of histone demethylases Utx and Uty in mouse brain and neurons. J Neurosci 2008, 28:4521-4527.

9. Dimitri P, Pisano C: Position effect variegation in Drosophila melanogaster: relationship between suppression effect and the amount of $Y$ chromosome. Genetics 1989, 122:793-800.

10. Meyer BJ: Targeting X chromosomes for repression. Curr Opin Genet Dev 2010, 20:179-189.

doi:10.1186/gb-2010-11-9-134

Cite this article as: Maatouk DM, Capel B: Unmasking a role for sex chromosomes in gene silencing. Genome Biology 2010, 11:134. 